\title{
A FAST MULTIPOLE METHOD FOR HIGHER ORDER VORTEX PANELS IN TWO-DIMENSIONS
}

\author{
PRABHU RAMACHANDRAN ${ }^{\dagger}$, S. C. RAJAN ${ }^{\ddagger}$, AND M. RAMAKRISHNA ${ }^{\ddagger}$
}

\begin{abstract}
Higher order panel methods are used to solve the Laplace equation in the presence of complex geometries. These methods are useful when globally accurate velocity or potential fields are desired as in the case of vortex based fluid flow solvers. This paper develops a fast multipole algorithm to compute velocity fields due to higher order, two-dimensional vortex panels. The technique is applied to panels having a cubic geometry and a linear distribution of vorticity. The results of the present method are compared with other available techniques.
\end{abstract}

Key words. Potential Flow, Fast Multipole Method, Higher Order Panels, Panel Method.

AMS subject classifications. 31A99, 34B60, 35J05, 65E05, 65Y99, 76M15

1. Introduction. Panel methods provide a means to solve incompressible and inviscid fluid flows in two and three dimensions. These methods reduce the dimensionality of the problem by one and hence in two dimensions require a one dimensional "grid" on the boundary. The flow of an inviscid and incompressible fluid in the presence of a solid body can be simulated by discretizing the body into elements called panels, distributing some singularity having an unknown strength on them and solving for the unknowns based on the boundary condition. In two dimensions the panel elements can be linear, parabolic or higher order. The singularity distributed can be a source, doublet or vorticity distribution. It can be lumped at a point or distributed as a constant, linear or higher order function. Katz and Plotkin [5] provide comprehensive details on panel methods in general. Hess and Smith [4 laid the foundation for the source panel method. The idea of the vortex panel method is due to Martensen 8 and is extended by Lewis 6 . Vortex panels unlike source panels can be used to simulate a lifting body. It is also well known that a polynomial distribution of doublets of order $q$ can be replaced by a vorticity distribution of order $q-1$. Therefore, the present work uses a vorticity distribution on the surface of the panels. Only two-dimensional flows are considered. It is to be noted that the ideas developed in this work can also be used for source and doublet distributions.

In panel methods, the boundary conditions can be represented in terms of the velocity resulting in the Neumann condition, or in terms of the potential or stream function on the boundary, called the Dirichlet condition. If $N$ panels with one unknown strength per panel are used to discretize the boundary, a system of $N$ linear equations is obtained based on the chosen boundary condition. The system of equations can be written as $\mathbf{A x}=\mathbf{b}$, where $\mathbf{x}$ represents the vector of $N$ unknown strengths. The boundary value problem is solved when $\mathbf{x}$ is obtained from these system of equations. In panel methods, $\mathbf{A}$ is usually dense. There are a variety of standard methods for the solution of dense matrices. These algorithms are usually computationally inefficient. For example, an LU decomposition requires $O\left(N^{3}\right)$ work. Rokhlin 12] uses a Generalized Conjugate Residual Algorithm (GCRA), ordinarily requiring $O\left(N^{2}\right)$ work, to solve the equations iteratively. In this algorithm one computes increasingly accurate approximations to $\mathbf{x}, \tilde{\mathbf{x}}$, by computing the matrix product $\mathbf{A} \tilde{\mathbf{x}}$. For well conditioned

\footnotetext{
${ }^{\dagger}$ Graduate Student, Department of Aerospace Engineering, IIT-Madras, Chennai 600036 (prabhu@aero.iitm.ernet.in).

${ }^{\ddagger}$ Department of Aerospace Engineering, IIT-Madras, Chennai 600036.
} 
matrices this sequence of approximations converges rapidly to $\mathbf{x}$. Normally, the matrix product A $\tilde{\mathbf{x}}$ involves $O\left(N^{2}\right)$ work. Rokhlin reduces this to an $O(N)$ computation by developing a fast multipole algorithm. This considerably speeds up the solution of the boundary value problem. Rokhlin developed the method for point sources/vortices (single layer potential) and doublets (double layer potential). These correspond to lumping the singularity distribution of the panel at a point. The resulting panels generate a singular velocity/potential field in the vicinity of these lumped points.

To eliminate the singular velocity or potential field, higher order distributions of singularity can be used. When linear panel elements are used with a vorticity distribution of any polynomial order $q \geq 1$, it can be seen [10, 11, that there is a logarithmic singularity at the edges of the panels. This is called the "edge effect". This edge effect can be eliminated by using a higher order panel method where the geometry of the panel is such that there is no discontinuity in the slope between two adjacent panels. For example, this is possible with cubic panels. The authors [10] developed a method that eliminates the edge effect by using cubic panel elements with a linear distribution of vorticity. This method was found to be about four times slower than the linear panel method when evaluating velocities or potentials for a given distribution of singularity. In [11 the authors use a fast multipole technique 3, 2, to accelerate the computation to acceptable speeds. The method uses a linear representation of panels for the far field fast multipole computations and uses the cubic method for the direct computations. While this hybrid approach eliminates the edge effect, it is not as accurate as the cubic panel method.

The present work details a procedure to perform a fast multipole summation using panels of any order and thus provides an accurate and fast technique for twodimensional panel methods. The method allows one to compute the matrix product A $\tilde{\mathbf{x}}$ in $O(N)$ time as done by Rokhlin [12] and thereby allows one to solve the boundary value problem efficiently. The technique is also of use when the velocity or potential field due to the panels having a known distribution of singularity is required at a very large number of points. Such a requirement arises in the context of vortex methods where the velocity field due to a collection of panels on a large number of vortex blobs is to be computed. The number of vortex blobs usually exceeds the number of panels by two orders of magnitude or more. In these cases it is possible to solve the boundary value problem using an LU decomposition since the number of panels involved is small. However, as mentioned in [11, p. 6], the computation of the velocity field due to the panels on the particles needs to be performed efficiently since the particle-particle interaction is computed using an $O(N)$ fast multipole method. The developed algorithm makes this possible.

The fast multipole method for higher order vortex panels developed in this work is demonstrated using cubic panels. It is compared with the hybrid algorithm described in 11] and also compared with Anderson's technique [1] extended to the current problem.

2. Mathematical details. For clarity, the velocity field due to a cubic panel element is first derived. Subsequently, the details of the fast multipole method are elaborated.

2.1. Cubic panels. Consider an arbitrarily oriented cubic panel as shown in figure 2.1. The start and end points of the panel are denoted as $z_{1}$ and $z_{2}$ respectively. The panel can be translated and rotated such that the start point is at the origin and the chord is along the $x$-axis in the $z^{\prime}$ plane. If the complex velocity due to the panel 


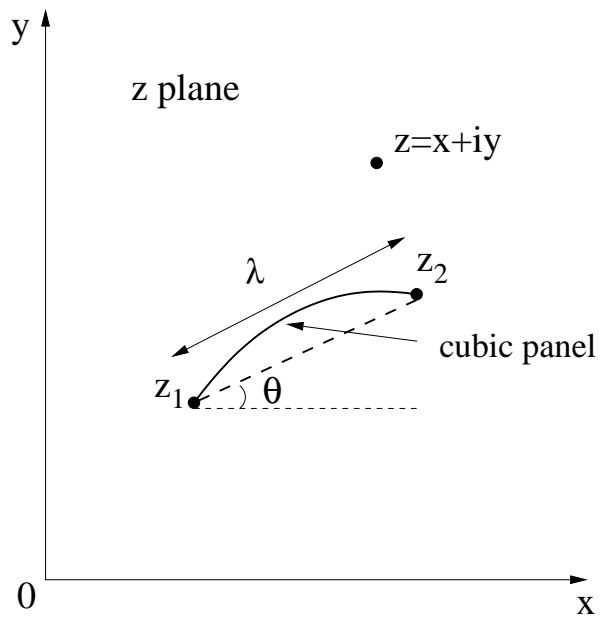

FIG. 2.1. Sketch of a single cubic panel having a chord length $\lambda$ in the $z$ plane.

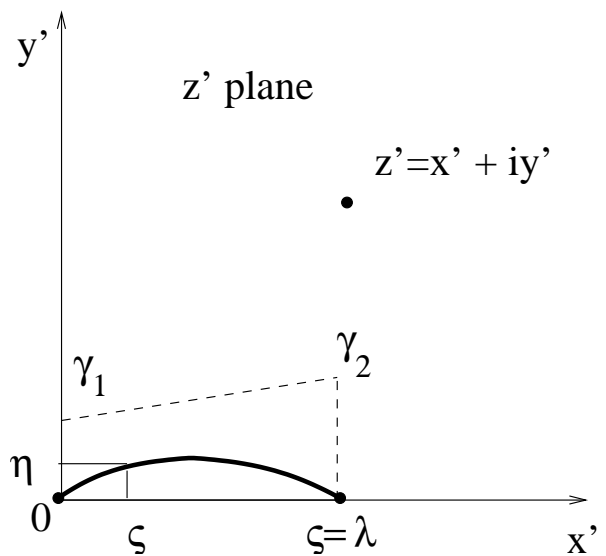

FIG. 2.2. Sketch of a single cubic panel having a chord length $\lambda$ in the $z^{\prime}$ plane.

in the $z^{\prime}$ plane is $V\left(z^{\prime}\right)$, then it is easy to see that the velocity, $V(z)$, in the $z$ plane can be obtained as, $V(z)=V\left(z^{\prime}\right) e^{-i \theta}$ and that $z=z_{1}+z^{\prime} e^{i \theta}$.

The equation of a cubic panel starting at the origin, with chord oriented along the $x$-axis is given by $\eta=a_{1} \zeta+a_{2} \zeta^{2}+a_{3} \zeta^{3}$, where $\zeta$ and $\eta$ respectively are the $x$ and $y$ coordinates of the panel surface, as shown in figure 2.2 Note that $0 \leq \zeta \leq \lambda$, where $\lambda$ is the chord length of the panel. The vorticity is distributed on the surface of the panel and is linear with respect to $\zeta$. The equation for the velocity at a point $z^{\prime}$ due to such a panel is given as

$$
V\left(z^{\prime}\right)=\frac{k}{2 \pi a_{3}} \int_{0}^{\lambda} \frac{\left(\zeta+\gamma_{1} / k\right)}{\left(\zeta^{3}+\frac{a_{2}}{a_{3}} \zeta^{2}+\frac{\left(a_{1}-i\right)}{a_{3}} \zeta+\frac{i z^{\prime}}{a_{3}}\right)} d \zeta
$$

where $k=\left(\gamma_{2}-\gamma_{1}\right) / \lambda$. As done in [11], the cubic in the denominator is factored as follows

$$
\zeta^{3}+\frac{a_{2}}{a_{3}} \zeta^{2}+\frac{\left(a_{1}-i\right)}{a_{3}} \zeta+\frac{i z^{\prime}}{a_{3}}=(\zeta-a)(\zeta-b)(\zeta-c)
$$


where $a, b$ and $c$ are the complex cube roots of the cubic 9. Given the roots, closed form integrals are obtained for the panel velocity and potential. After integration and simplification the velocity due to the cubic panel in the $z$ plane is,

$$
\begin{aligned}
V(z)= & \frac{-\gamma_{2}}{2 \pi a_{3} \lambda}\left[\frac{a \log \left(\frac{a-\lambda}{a}\right)}{(a-c)(a-b)}+\frac{b \log \left(\frac{b-\lambda}{b}\right)}{(b-c)(b-a)}+\frac{c \log \left(\frac{c-\lambda}{c}\right)}{(c-a)(c-b)}\right] e^{-i \theta} \\
& -\frac{\gamma_{1}}{2 \pi a_{3} \lambda}\left[\frac{(\lambda-a) \log \left(\frac{a-\lambda}{a}\right)}{(a-c)(a-b)}+\frac{(\lambda-b) \log \left(\frac{b-\lambda}{b}\right)}{(b-c)(b-a)}+\frac{(\lambda-c) \log \left(\frac{c-\lambda}{c}\right)}{(c-a)(c-b)}\right] e^{-i \theta},
\end{aligned}
$$

where $\theta$ is the angle between the chord of the cubic panel and the $x$-axis. Similarly, the complex potential due to the panel can be obtained. The expressions for these are not reproduced here. Using these derived expressions one can compute the velocity or potential due to a cubic panel with a linear vorticity distribution. It it to be noted that if the panel geometry is almost parabolic $\left(a_{3} \rightarrow 0\right)$ or linear $\left(a_{3}, a_{2} \rightarrow 0\right)$, the above expression for the velocity field would be inaccurate. In such cases, the panels should be represented as parabolic or linear elements and the velocity integral specialized appropriately. These are straightforward to derive. The specialization does not affect the developed method in any way.

2.2. FMM for higher order panels. Consider a higher order panel with its chord oriented along the $\mathrm{x}$-axis as illustrated in figure 2.2 . Given a vorticity distribution $\gamma(\zeta)$ it can be easily seen that the complex velocity at a point $z^{\prime}$ due to the panel is given as,

$$
V\left(z^{\prime}\right)=u^{\prime}-i v^{\prime}=\frac{-i}{2 \pi} \int_{0}^{\lambda} \frac{\gamma(\zeta)}{z^{\prime}-(\zeta+i \eta)} d \zeta .
$$

Substituting $\xi=\zeta+i \eta$, and performing a binomial expansion results in,

$$
\begin{aligned}
u^{\prime}-i v^{\prime} & =\frac{-i}{2 \pi} \int_{0}^{\lambda} \frac{\gamma(\zeta)}{z^{\prime}-\xi} d \zeta \\
& =\frac{-i}{2 \pi} \sum_{j=1}^{\infty} \int_{0}^{\lambda} \frac{\gamma(\zeta) \xi^{j-1}}{z^{\prime j}} d \zeta
\end{aligned}
$$

Without loss of generality, if $z_{1}$ of the panel is assumed to be at the origin, then $z^{\prime}=z e^{-i \theta}$ and $u-i v=e^{-i \theta}\left(u^{\prime}-i v^{\prime}\right)$, and the above equation reduces to,

$$
u-i v=\frac{-i}{2 \pi} \sum_{j=1}^{\infty} \frac{e^{i(j-1) \theta}}{z^{j}} \int_{0}^{\lambda} \gamma(\zeta) \xi(\zeta)^{j-1} d \zeta .
$$

This can be written as,

$$
u-i v=\frac{-i}{2 \pi} \sum_{j=1}^{\infty} \frac{A_{j}}{z^{j}},
$$

where,

$$
A_{j}=e^{i(j-1) \theta} \int_{0}^{\lambda} \gamma(\zeta) \xi(\zeta)^{j-1} d \zeta
$$


The complex potential of the higher order panel can also be obtained as follows,

$$
\begin{aligned}
\Phi=\phi+i \psi & =\frac{-i}{2 \pi} \int_{0}^{\lambda} \gamma \ln \left(z^{\prime}-\xi\right) d \zeta \\
& =\frac{-i}{2 \pi}\left(\ln \left(z^{\prime}\right) \int_{0}^{\lambda} \gamma d \zeta-\sum_{k=1}^{\infty} \frac{1}{z^{\prime k}} \int_{0}^{\lambda} \frac{\xi^{k}}{k} d \zeta\right) \\
& =\frac{-i}{2 \pi}\left[P_{0} \ln \left(z^{\prime}\right)-\sum_{k=1}^{\infty} \frac{P_{k}}{z^{\prime k}}\right]
\end{aligned}
$$

where,

$$
P_{0}=\int_{0}^{\lambda} \gamma d \zeta ; \quad P_{k}=\frac{1}{k} \int_{0}^{\lambda} \xi^{k} \gamma d \zeta .
$$

In the present work we are interested in evaluating the velocity fields. Hence, the multipole method is developed with that in mind. The analysis of the truncation errors is performed only for the velocity field. The truncation error for the complex potential can also be easily computed in a similar fashion as done for the velocity field.

Given $\xi(\zeta), \gamma(\zeta)$ and $\theta, A_{j}$ can be readily computed. The series (2.7) converges if $|\xi|<|z|$. It is reasonable to assume that the panel is completely contained inside a circle centered at the origin having radius $\lambda$, i.e. $|\xi(\zeta)|<\lambda$. Given this, it is easy to see from (2.8) that the error involved in truncating the series to a finite number of terms $p$ is,

$$
\left|V(z)+\frac{i}{2 \pi} \sum_{j=1}^{p} \frac{A_{j}}{z^{j}}\right| \leq \frac{\Gamma}{2 \pi \lambda(\varrho-1)}\left(\frac{1}{\varrho}\right)^{p}
$$

where,

$$
\Gamma=\int_{0}^{\lambda}|\gamma(\zeta)| d \zeta
$$

and $\varrho=|z| / \lambda$.

Hence, equations (2.7) and (2.8) can be used to obtain a fast multipole expansion for higher order panels. The coefficients $A_{j}$ are to be computed by numerical integration. It is to be noted that equation (2.7) is a multipole expansion about the point $z_{1}$ (the first point) of the panel as shown in figure 2.1. For a cubic panel as used in the earlier work [11] we have, $\xi=\zeta+i\left(a_{1} \zeta+a_{2} \zeta^{2}+a_{3} \zeta^{3}\right)$. It is also to be noted that if the panels do not deform or change orientation, the coefficients, $A_{j}$, are constant and need be computed only once. If the panels only rotate, the entire integral need not be evaluated and the coefficients need to be multiplied by a different value of $e^{i(j-1) \theta}$. If the panels deform, the coefficients must be recomputed. Given equations (2.7) and (2.8), the various expressions for the fast multipole method can be derived as follows.

2.3. Multipole expansion for a collection of panels. Given $n$ panels placed at points $z_{k}$ inside a circle of radius $R$, the multipole expansion for the velocity field of the panels is,

$$
V(z)=u-i v=\frac{-i}{2 \pi} \sum_{k=1}^{n} \sum_{j=1}^{\infty} \frac{A_{k j}}{\left(z-z_{k}\right)^{j}}
$$


where $A_{k j}$ are the coefficients as given in equation (2.8) for the panel starting at $z_{k}$. The above can be expressed as a multipole expansion about a circle centered at $z_{0}$ as follows,

$$
V(z)=\frac{-i}{2 \pi} \sum_{k=1}^{n} \sum_{j=1}^{\infty} \frac{A_{k j}}{\left(z-z_{0}\right)^{j}}\left[1-\left(z_{k}-z_{0}\right) /\left(z-z_{0}\right)\right]^{-j} .
$$

By grouping powers of $\left(z-z_{0}\right)$ it can be seen that,

$$
V(z)=\frac{-i}{2 \pi} \sum_{j=1}^{\infty} \frac{a_{j}}{\left(z-z_{0}\right)^{j}}
$$

where,

$$
a_{j}=\sum_{k=1}^{n} \sum_{m=1}^{j} A_{k m}\left(\begin{array}{c}
j-1 \\
m-1
\end{array}\right)\left(z_{k}-z_{0}\right)^{j-m}
$$

Equations (2.13) and (2.14) are equivalents to those of Lemma 2.1 in Carrier et al. 2]. This expression is nothing but the sum of the transfers of the multipole expansions of the $n$ panels, each starting at $z_{k}$, to a circle of radius $R$ centered at $z_{0}$. Thus, when this expression is truncated to $p$ terms, the error in the velocity is,

$$
\left|V(z)+\frac{i}{2 \pi} \sum_{j=1}^{p} \frac{a_{j}}{\left(z-z_{0}\right)^{j}}\right|=\left|\frac{i}{2 \pi} \sum_{j=p+1}^{\infty} \frac{a_{j}}{\left(z-z_{0}\right)^{j}}\right|,
$$

where $a_{j}$ is given in equation (2.14). From equation $(\overline{2.8})$ it can be seen that,

$$
\left|A_{k m}\right| \leq \Gamma_{k} \lambda_{k}^{m-1}, \quad \Gamma_{k}=\int_{0}^{\lambda_{k}}\left|\gamma_{k}(\zeta)\right| d \zeta
$$

where $\lambda_{k}$ is the chord length of the $k^{\prime}$ th panel. Given this and the fact that all the $z_{k}$ 's lie inside a circle of radius $R, a_{j}$ can be clearly bounded as,

$$
\begin{aligned}
\left|a_{j}\right| & \leq \sum_{k=1}^{n} \sum_{m=1}^{j} \Gamma_{k} \lambda_{k}^{m-1}\left(\begin{array}{c}
j-1 \\
m-1
\end{array}\right) R^{j-m} \\
& \leq \Delta \sum_{m=1}^{j} \lambda^{m-1}\left(\begin{array}{c}
j-1 \\
m-1
\end{array}\right) R^{j-m}
\end{aligned}
$$

where $\Delta=\sum_{k=1}^{n} \Gamma_{k}$ and $\lambda=\max \left(\lambda_{k}\right)$. Hence,

$$
\left|a_{j}\right| \leq \Delta(R+\lambda)^{j-1}
$$

Therefore,

$$
\begin{aligned}
\left|V(z)+\frac{i}{2 \pi} \sum_{j=1}^{p} \frac{a_{j}}{\left(z-z_{0}\right)^{j}}\right| & \leq\left|\frac{i \Delta}{2 \pi} \sum_{j=p+1}^{\infty} \frac{(R+\lambda)^{j-1}}{\left(z-z_{0}\right)^{j}}\right|^{p} \\
& =\frac{\Delta}{2 \pi(R+\lambda)(c-1)}\left(\frac{1}{c}\right)^{p}
\end{aligned}
$$




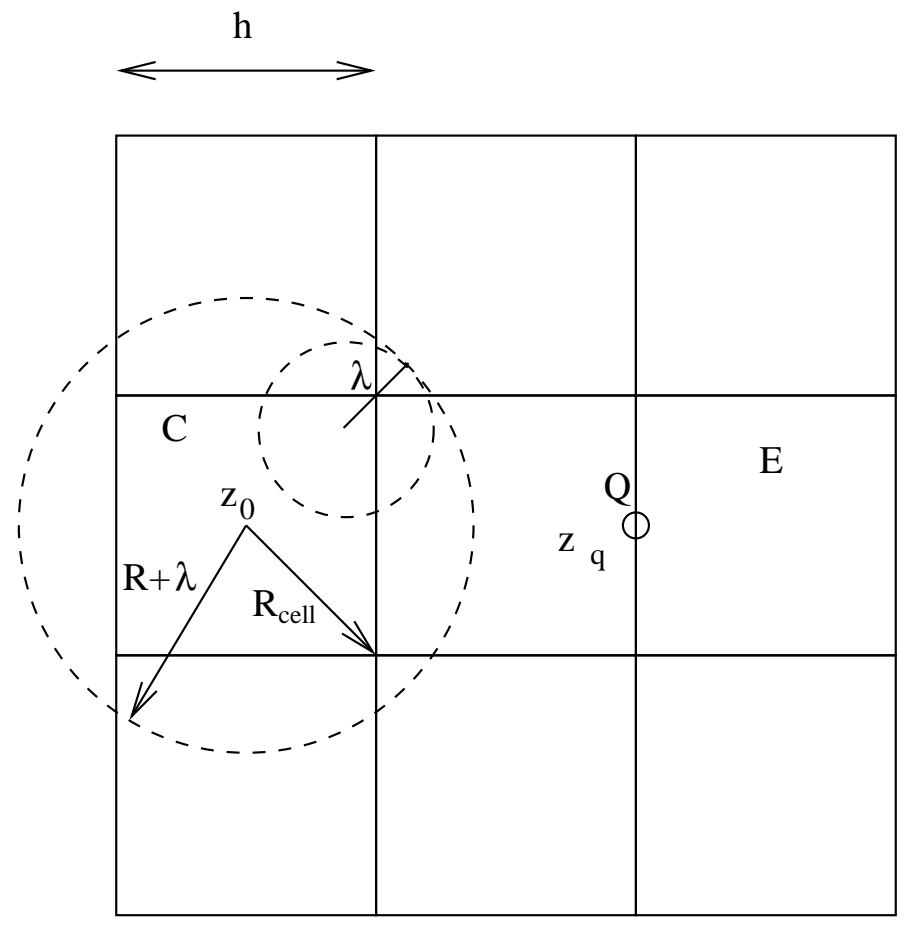

FIG. 2.3. Illustration of radius of convergence for panels.

where $c=\left|z-z_{0}\right| /(R+\lambda)$. It is to be noted that in this case, any panel having a starting point $z_{k}$, lying inside the circle $D$, centered at $z_{0}$ with radius $R$, is considered for the multipole expansion. Clearly, the circle of radius $R+\lambda$ will completely contain all the panels that have a starting point inside $D$. Let the radius of a multipole cell containing panels be $R_{\text {cell }}$. If the panel mid-chord position is used as the determining criterion for a panel to be part of a cell, then clearly the radius of the circle that completely contains all the panels in that cell is $R_{\text {cell }}+\lambda / 2$. This is because only half a panel length will be partly outside the cell. From this it is easy to see that

$$
R+\lambda \leq R_{\text {cell }}+\lambda / 2
$$

While the result in equation (2.16) has the same form $\left(O\left(c^{-p}\right)\right)$ for the error term as in the fast multipole method of [3 2], the value of $c$ is different. The reason for the difference can be explained as follows. Consider the cell $C$ centered at $z_{0}$, shown in figure 2.3 . The cell contains a panel of length $\lambda$, with its mid point at the corner of the cell and at an angle $45^{\circ}$ to the horizontal. The size of the cell is $h$. The radius of the cell $R_{\text {cell }}=h \sqrt{2} / 2$. From equation (2.11) it is clear that the panel's multipole expansion about its start point converges only outside a circle of radius $\lambda$ centered about its starting point. Thus, it is easy to see that the multipole expansion for the cell about its center converges outside a circle of radius $R_{\text {cell }}+\lambda / 2$. This is the same result as shown in equation (2.17).

When implementing the adaptive fast multipole algorithm, the multipole expansion of the cell $C$ can be evaluated on cells that are well separated from it. The cell $E$ contains $z_{q}$, the closest point to $z_{0}$. The error in the multipole expansion at this 
point is governed by,

$$
c=\left|\frac{z_{q}-z_{0}}{R+\lambda}\right|=\frac{3 h}{h \sqrt{2}+\lambda}
$$

If $h=\beta \lambda$, then,

$$
c=\frac{3 \beta}{\beta \sqrt{2}+1}
$$

$c$ is not always greater than 2 as in the case of the original fast multipole method where $c=3 / \sqrt{2}$. Given a value of $c$ and a desired precision, $\epsilon$, the number of terms in the series necessary, $p$, can be determined from equation (2.16). In order to employ a smaller number of terms $p, \beta$ needs to be chosen carefully. It is important to note that the size of the cells used in the multipole method should be limited by $\beta \lambda$. This result also applies to the fast multipole method of [11.

2.4. Shifting the center of a multipole expansion. Equation (2.13) is the multipole expansion for a collection of panels in a circle of radius $R$ centered at $z_{0}$. This expansion, when translated to the origin, produces a multipole expansion that converges outside a circle centered at the origin with radius $R+\lambda+\left|z_{0}\right|$. The resulting multipole expansion is given by

$$
V(z)=\frac{-i}{2 \pi} \sum_{j=1}^{\infty} \frac{b_{j}}{z^{j}}
$$

where,

$$
b_{j}=\sum_{k=1}^{j} a_{k}\left(\begin{array}{c}
j-1 \\
k-1
\end{array}\right) z_{0}^{j-k}
$$

and $a_{k}$ is as given in equation (2.14). This expression is the equivalent of Lemma 2.2 in [2]. From equation (2.15) a bound for $b_{j}$ can be obtained as,

$$
\begin{aligned}
\left|b_{j}\right| & \leq \Delta \sum_{k=1}^{j}(R+\lambda)^{k-1}\left(\begin{array}{l}
j-1 \\
k-1
\end{array}\right)\left|z_{0}\right|^{j-k} \\
& =\Delta\left(R+\lambda+\left|z_{0}\right|\right)^{j-1}
\end{aligned}
$$

From the above, the error in truncating equation (2.19) to $p$ terms is,

$$
\begin{aligned}
\left|V(z)+\frac{i}{2 \pi} \sum_{j=1}^{p} \frac{b_{j}}{z^{j}}\right| & =\left|\frac{i}{2 \pi} \sum_{j=p+1}^{\infty} \frac{b_{j}}{z^{j}}\right| \\
& \leq \frac{\Delta}{2 \pi\left(|z|-\left(R+\lambda+\left|z_{0}\right|\right)\right)}\left(\frac{R+\lambda+\left|z_{0}\right|}{|z|}\right)^{p}
\end{aligned}
$$

2.5. Conversion of multipole expansion to a local expansion. Given a multipole expansion (2.13) about a circle $D_{0}$ of radius $R$ and centered at $z_{0}$ such that $\left|z_{0}\right|>(c+1) R$ with $c>1$, the multipole expansion can be described by a power series in $z$ that converges inside a circle, $D_{2}$, centered at the origin having radius $R$,

$$
V(z)=\sum_{j=0}^{\infty} c_{j} z^{j}
$$




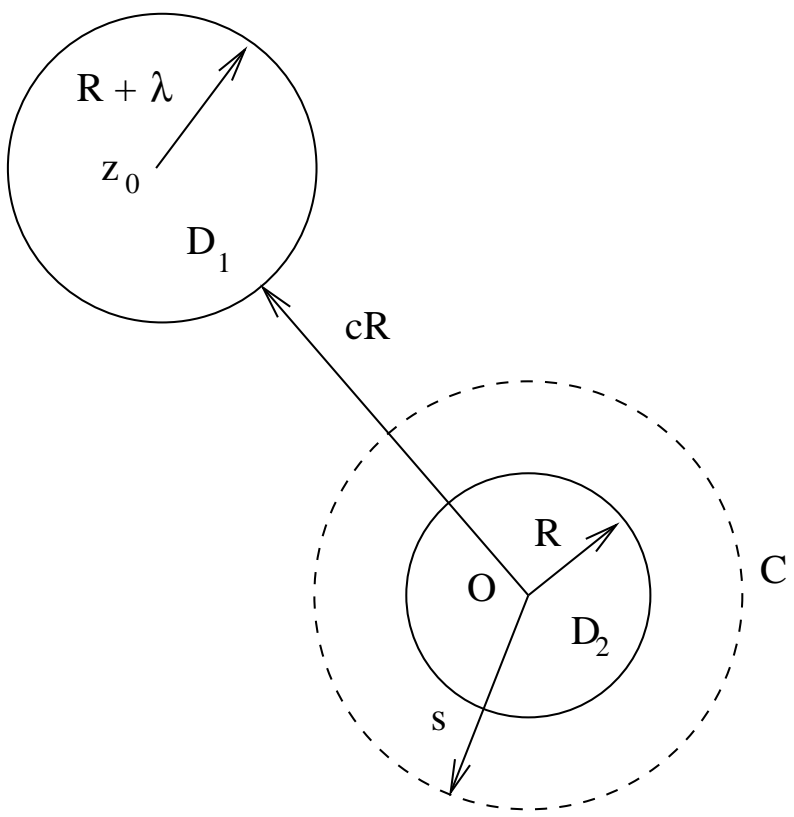

FIG. 2.4. Illustration for the truncation error involved in evaluating a local expansion.

where,

$$
c_{j}=\frac{-i}{2 \pi z_{0}^{j}} \sum_{k=1}^{\infty}(-1)^{k} \frac{a_{k}}{z_{0}^{k}}\left(\begin{array}{c}
j+k-1 \\
k-1
\end{array}\right) .
$$

This is the equivalent of Lemma 2.3 in [2]. The derivation for the error term is similar to that derived in [3] for the local expansions. However, the extent of the panels modifies the results slightly. In the following, the error introduced when the series in equation (2.23) is truncated to $p$ terms is derived. The derivation is very similar to that in [3].

Consider figure 2.4. The multipole expansion about the center of the circle $D_{0}$ is transferred to the center of circle $D_{2}$ as a local expansion about the origin. Although the circle $D_{0}$ is of radius $R$, we consider a circle $D_{1}$ of radius $R+\lambda$ because the multipole expansion for the panels will converge for a point outside the larger circle. The circle $C$ has radius $s$. From Taylor's theorem it is known that,

$$
\left|c_{j}\right| \leq \frac{M}{s^{j}}
$$

where $M=\max (|V(z)|)$ on the circle $C . s$ is defined as per Greengard and Rokhlin's paper [3] as $s=c R((p-1) / p)$. When $p \geq 2 c /(c-1)$

$$
R<c(R+1) / 2<s<c R \text {. }
$$

If $t$ is a co-ordinate along the circumference of $C$ it is easy to see that $\left|t-z_{0}\right| \geq$ $R(C+1)+\lambda-s$. Hence, from equations (2.15), (2.15) and the definition for $s$, we have that,

$$
M=\max (|V(t)|) \leq \frac{1}{2 \pi} \sum_{j=1}^{\infty} \frac{\left|a_{j}\right|}{\left|t-z_{0}\right|^{j}} \leq \frac{\Delta(p(R+\lambda)+c R)}{2 \pi(R+\lambda) c R}
$$


Hence, the truncation error is given by,

$$
\begin{aligned}
\left|V(z)-\sum_{j=1}^{p} c_{j} z^{j}\right| & \leq \sum_{j=p+1}^{\infty}\left|c_{j}\right||z|^{j} \\
& \leq \frac{\Delta(p(R+\lambda)+c R)}{2 \pi(R+\lambda) c R}\left|\frac{z}{s}\right|^{p+1} \frac{1}{1-\frac{|z|}{s}}
\end{aligned}
$$

If $p \geq 2 c /(c-1)$ then it can be shown that,

$$
1-\frac{|z|}{s} \geq \frac{c-1}{c+1}
$$

From the definition of $s$ it can be seen that,

$$
\left|\frac{z}{s}\right|^{p+1} \leq\left(\frac{1}{c}\right)^{p+1}\left(1+\frac{1}{p-1}\right)^{p-1}\left(1+\frac{1}{p-1}\right)^{2}
$$

Clearly, when $p \geq 2$ this reduces to

$$
\left|\frac{z}{s}\right|^{p+1} \leq 4 e\left(\frac{1}{c}\right)^{p+1}
$$

where $e$ is the base of the natural logarithm. Hence, for any $p \geq \max (2,2 c /(c-1))$ it is easy to see that,

$$
\left|V(z)-\sum_{j=1}^{p} c_{j} z^{j}\right| \leq \frac{2 \Delta e(p(R+\lambda)+c R)(c+1)}{\pi c R(c-1)(R+\lambda)}\left(\frac{1}{c}\right)^{p+1}
$$

This expression is very similar to that in 3 . Also note that $c$ is as defined in 3 and is not a function of $\lambda$.

2.6. Shifting the center of a local expansion. Given any local expansion centered about $z_{0}$ and the coefficients $a_{k}$ the center of the local expansion can be shifted to the origin using,

$$
\sum_{i=0}^{n} a_{i}\left(z-z_{0}\right)^{i}=\sum_{j=0}^{n} z^{j} \sum_{k=j}^{n} a_{k}\left(\begin{array}{c}
k \\
j
\end{array}\right)\left(-z_{0}\right)^{k-j}
$$

This expression is exact and is the same as that of Lemma 2.4 from [2].

Using equations $(2.13),(2.19),(2.23)$ and $(2.26)$ the fast multipole algorithm can be applied to higher order panels. As detailed in section 2.3 , it is to be noted that the size of the cell is limited by the length of the panels and the parameter $c$ in equation $(2.16)$ is modified as in equation (2.18). The direct computation of the velocity is dependent on the actual higher order panel chosen. In the present work a cubic panel geometry with a linear distribution of vorticity is used. The direct computation of the velocity field due to this type of panel is performed using equation (2.3).

3. Anderson's FMM without multipoles. The results of the present method are compared with those obtained using Anderson's[1] technique applied to the cubic panels. This technique is called "FMM without multipoles". This method uses 
Poisson's integral formula in order to obtain equivalents for the multipole expansion. Given a collection of point charges or vortices the method uses inner and outer ring approximations of the potential, that are computed using Poisson's integral formula, to represent the cluster of particles as a single computational entity and accelerate the computations. The advantage of this approach is that there is no need to obtain expressions for the multipole expansions as done normally with the FMM. Anderson describes the algorithm and its implementation from a multigrid perspective. The present work translates the algorithm from the multigrid implementation to that of an adaptive fast multipole algorithm. The various lists $\left(U_{b}, V_{b}, W_{b}, X_{b}\right.$ and $\left.Y_{b}\right)$ and other symbols used in the discussion are the same as those described and used in 22. The translation is simple and is performed as follows.

- The multipole expansions for the childless cells are computed using outer ring approximations of the potential.

- The multipole expansions from daughter cells, $D$, are are shifted to their parents, $P$, by evaluating the outer ring approximation of $D$ on $P$ 's outer integration points and accumulating the outer ring coefficients of $P$.

- Given a multipole expansion (or an outer ring approximation) of a cell $C$, a local expansion (inner ring approximation) at a well separated cell $S$, can be obtained by computing the outer ring approximation of the velocity/potential due to $C$ on $S$ 's inner ring integration points and adding them to the inner ring coefficients of $S$. This corresponds to obtaining the interactions due to cells in the $V_{b}$ list as described in [2].

- A local expansion is translated from a cell $P$, to a child cell $C$, by evaluating the inner ring approximation of $P$ at the inner integration points of $C$ and adding the result to the inner ring approximation coefficients of $C$.

- The interactions due to a cell $W$, in the $W_{b}$ list of a cell $B$ are computed by evaluating the velocity/potential due to the outer ring of $W$ on particles inside $B$.

- The $\Delta_{b}$ interactions on cell $B$ due to a cell $X$ belonging to the $X_{b}$ list is found by evaluating the potential due to each panel in $X$ on the inner ring integration points of $B$.

The algorithm does not require the use of explicit expressions for the multipole expansions and the local expansions. This makes the method easy to extend to situations where expressions for the multipole expansion are not easy to derive.

Since the focus of this work is to compute the velocity field of panels, the expressions for the potential in Anderson's work [1] are differentiated to obtain the velocity directly rather than by numerical differentiation. It is to be noted that in the case of vortex blobs or vortex panels, it is the stream function and not the potential that behaves like $\log (r)$. Thus, the stream function of the panels must be used in the present computations.

There are a few important issues to keep in mind when using Anderson's scheme. The outer ring approximation for the stream function due to a collection of panels inside a cell is given as,

$$
\psi(r, \theta) \approx \kappa \log (r)+\frac{1}{2 \pi} \sum_{i=1}^{K} f\left(s_{i}\right) F_{i} h
$$




$$
F_{i}=\frac{1-\left(\frac{a}{r}\right)^{2}-2\left(\frac{a}{r}\right)^{M+1} \cos \left((M+1)\left(\theta-s_{i}\right)\right)+2\left(\frac{a}{r}\right)^{M+2} \cos \left(M\left(\theta-s_{i}\right)\right)}{1-2\left(\frac{a}{r}\right) \cos \left(\theta-s_{i}\right)+\left(\frac{a}{r}\right)^{2}}
$$

where, $r, \theta$ are the co-ordinates of a point where the stream function is approximated. $K=2 M+1, s_{i}$ is the angle of the integration point on the ring of radius $a, h=2 \pi a / K$, $\kappa=\sum_{i=1}^{N}\left(\kappa_{i} / 2 \pi\right), f\left(s_{i}\right)=\Psi\left(a, s_{i}\right)-\kappa \log (a)$ and $\Psi$ is the stream function induced by $N$ vortex panels of strengths $\kappa_{i}$. The inner ring approximation is similar and given as,

$$
\begin{aligned}
\psi(r, \theta) & \approx \frac{1}{2 \pi} \sum_{i=1}^{K} f\left(s_{i}\right) G_{i} h \\
G_{i} & =\frac{1-\left(\frac{r}{a}\right)^{2}-2\left(\frac{r}{a}\right)^{M+1} \cos \left((M+1)\left(\theta-s_{i}\right)\right)+2\left(\frac{r}{a}\right)^{M+2} \cos \left(M\left(\theta-s_{i}\right)\right)}{1-2\left(\frac{r}{a}\right) \cos \left(\theta-s_{i}\right)+\left(\frac{r}{a}\right)^{2}}
\end{aligned}
$$

where, $f\left(s_{i}\right)$ is the stream function induced by particles outside the inner ring on the inner ring. For a ball of radius $R$, containing a collection of particles (or panels) Anderson suggests using a value of $a=2 R$ for the outer ring approximation. For an inner ring approximation the value of $a=R / 2$ can be chosen.

The expression for $F_{i}$ and $G_{i}$ in equations (3.1) and (3.2) are indeterminate when the evaluation point is at any of the integration points, i.e. when $r=a$ and $\theta=s_{i}$. However, the functions are very well behaved and it can be shown that

$$
\lim _{r \rightarrow a ; \theta \rightarrow s_{i}} F_{i}=\lim _{r \rightarrow a ; \theta \rightarrow s_{i}} G_{i}=2 M+1 .
$$

It is to be noted that the limiting value is the same independent of the order in which the limits are taken.

In the present case we are interested in the evaluation of the velocity field and not the stream function. In order to obtain the velocity field one can differentiate equations (3.1) and (3.2). From the expressions for $G_{i}, F_{i}$ and the equation (3.3) it can be shown that the derivatives of $F_{i}$ and $G_{i}$ will be singular at the integration points. If the inner ring lies inside a cell, the points at which the velocities are evaluated are likely to be near the integration points (i.e. $r \rightarrow a$ and $\theta \rightarrow s_{i}$ ). Hence, inaccurate results will be obtained for such points. Figure 3.1 plots the absolute error when a single panel in a cell of radius $R$ is considered. The outer ring approximation with $a=2 R$ is considered and the velocity is evaluated at 2000 points on the ring. Similarly, a well separated cell $S$, is considered and the outer ring approximation transferred to the inner ring of $S$ and the derivative of the inner ring approximation on $S$ is evaluated with $a=R / 2$ on 2000 points on the inner ring of $S$. As can be seen, when the angle $\theta$ is zero there is a very large error. This is because exactly at this point there is an integration point $\left(s_{i}=0\right)$. Similarly, very large errors are seen near the other evaluation points indicating that the derivatives of $F_{i}$ and $G_{i}$ diverge at the evaluation points. In order to avoid such problems, the ring radii must be chosen carefully. This must be done without loss of accuracy. The inner ring radius must be chosen such that it is outside the cell where the velocity is to be evaluated. The outer ring radius must be chosen such that it does not intersect any well separated cell. It is easy to see that the inner ring radius should be such that $a>R$ and for the outer ring it must be such that $a<3 R$. Anderson's choice 


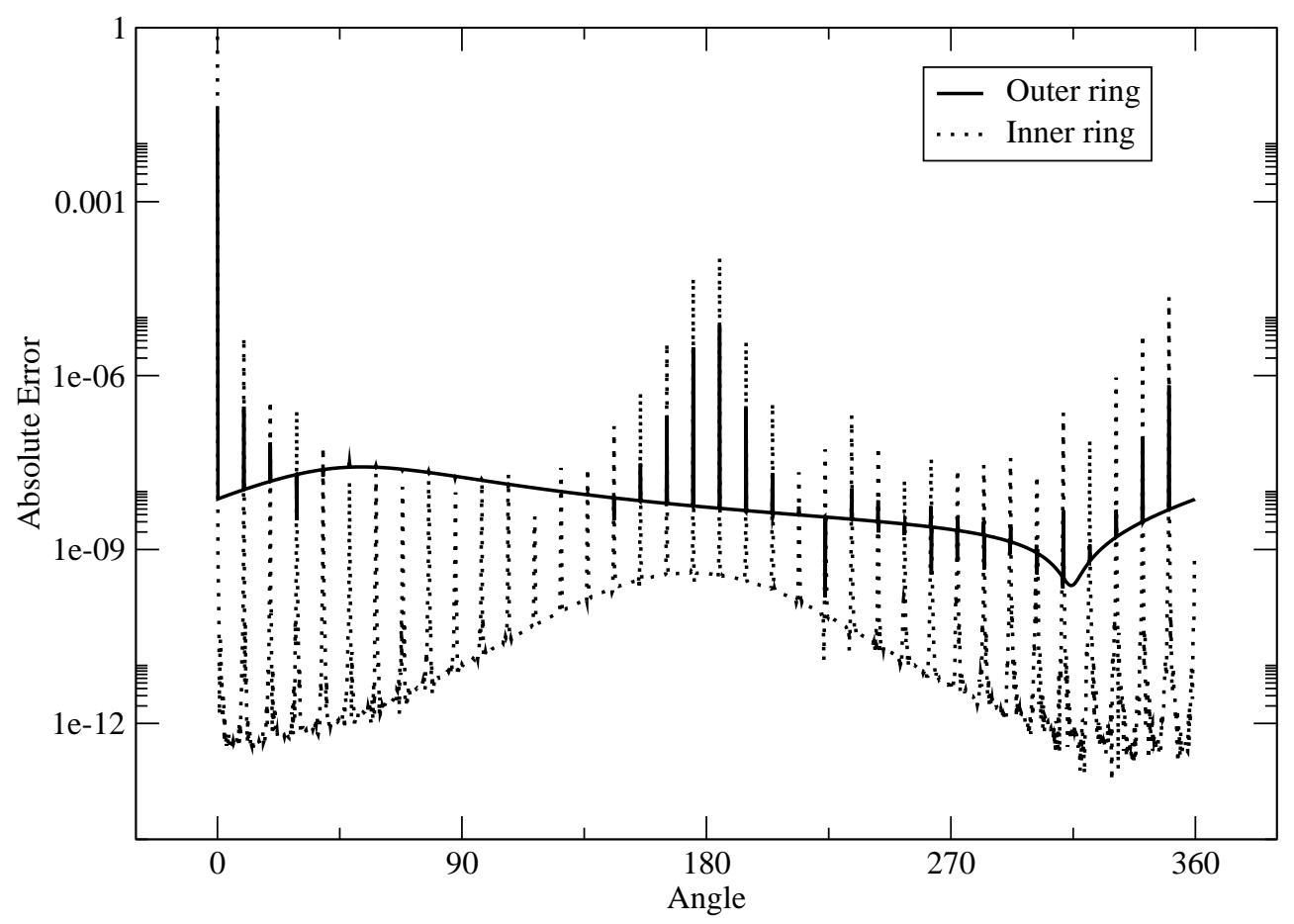

FIG. 3.1. Absolute error in the evaluation of velocity for points on the inner and outer rings. The velocity is obtained by differentiating the inner and outer ring approximations. $a=R / 2$ for the inner ring approximation and $a=2 R$ for the outer ring approximation. $R$ is the radius of the cell considered.

of the outer ring radius satisfies the present criterion. In the present case, given a cell of length $h$ and radius $R=h \sqrt{2} / 2$, it was found that choosing the $a=0.75 h$ for the inner ring radius works well without any significant loss of accuracy. In order to demonstrate this, a radial line starting from the center of the cell and extending out to $1.5 R$ is considered and the absolute error in the inner ring approximation of the velocity is plotted versus distance from the center. Figure 3.2 plots the absolute error versus relative distance from the center of the cell. As can be seen, there is very little loss of accuracy when the inner ring is moved outside the cell. A few bumps are seen in the plot for the $a=R / 2$ case when the relative distance is near 0.5 . This is because the evaluation points on the line pass very close to an integration point. Hence, by moving the inner ring outside the cell the possibility for error in evaluating the velocity due to the inner ring approximation near the integration points is eliminated. For the outer ring radius a value of $a=1.4 h$ is chosen. This ensures that the outer ring does not lie inside any well separated cells. In this manner it is possible to obtain accurate velocity fields using Anderson's technique. It is to be noted that the above inner ring radius should be used even if the pseudo-particle multipole method of Makino [7] is used. This is because the pseudo-particle multipole method uses Anderson's expressions for the local expansions. The work of Vosbeek et al. [13. eliminates the need for this change in the inner ring radius. Instead of dealing with 


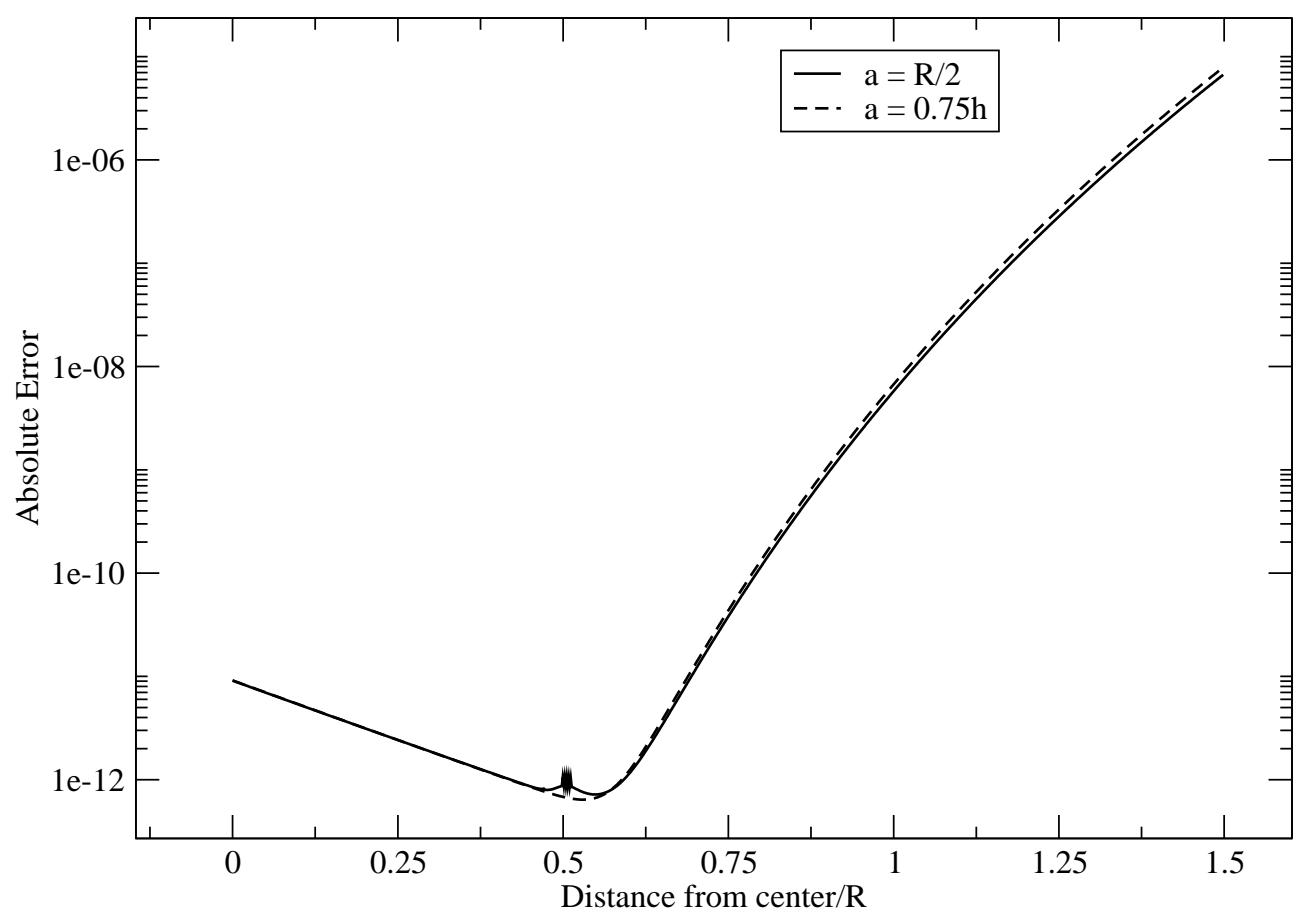

FIG. 3.2. Absolute error in the velocity evaluation due to an inner ring approximation for points along a radial line. The plots for $a=R / 2$ and $a=0.75 h$ are shown.

the potential/stream function and differentiating it analytically or numerically they re-derive the expressions for the components of the velocity field $\left(u_{r}\right.$ and $\left.u_{\theta}\right)$ in terms of Poisson's integral and thus eliminate the problems in the vicinity of the evaluation points. At the evaluation points the expressions for the velocity are indeterminate. However, the functions are well behaved in the neighborhood of these points and one can use the limiting value of the function. Hence, their technique could also be used to compute the velocity. Anderson's scheme requires only the potential/stream function to be computed whereas their scheme requires handling both components of the velocity. In the present work we have chosen to use Anderson's scheme for its simplicity.

4. Implementation details. The expressions derived in section 2 can be easily incorporated in an existing fast multipole algorithm implementation. The implementation of the fast multipole algorithm itself is standard and described in Carrier et al. 2]. Other issues specific to the present implementation are detailed in this section.

In subsequent discussions it is to be noted that a "cell" refers to a box of the adaptive mesh. The cell containing all particles and panels is at level 0. Cells at level $l+1$ are obtained by splitting a cell at level $l$ into four daughter cells. A parent cell is one that has children and a childless cell is a cell that is not a parent. A colleague of a cell $c$ is defined as a cell at the same level as that of $c$ and having a shared side or corner with it. 
In the present work the domain decomposition for both the new method and Anderson's scheme are performed as described in Ramachandran et al. [11. This scheme handles tracer particles efficiently in the context of the FMM. The basic idea being that the particles are classified into "causes" and "effects". The causes influence the effects. The cell splitting is controlled depending on the number of causes and effects in a cell and in its colleagues. Numerical experiments are performed in order to compute the maximum number of cause and effect particles per cell.

The expression for the multipole coefficients for a panel in equation (2.8) seems to indicate that the multipole coefficients need re-computation when the value of $\gamma(\zeta)$ changes. For a linear distribution of vorticity the expression for $A_{j}$ is given below,

$$
A_{j}=e^{i(j-1) \theta}\left(\gamma_{1} \int_{0}^{\lambda} \xi(\zeta)^{j-1} d \zeta+\frac{\gamma_{2}-\gamma_{1}}{\lambda} \int_{0}^{\lambda} \zeta \xi(\zeta)^{j-1} d \zeta\right)
$$

where $\gamma_{1}$ and $\gamma_{2}$ are the values of the vorticity at the ends of the panel. Clearly the integrals are independent of $\gamma_{1}$ and $\gamma_{2}$ and need not be re-computed unless the geometry of the panel or its orientation change. In the present work the integrals in the equation are evaluated using Simpson's rule.

At the end of section 2.3 it was mentioned that in order to obtain accurate results the cell size needs to be limited based on the panel size. This can be done efficiently in the following manner. The maximum and minimum panel lengths $\left(\lambda_{\max }, \lambda_{\min }\right)$ are computed when assigning the panels to the cell at level 0 . If there is a significant length variation in the panels, then the $\lambda_{\max }$ at higher levels must be recomputed. If not, the same value of $\lambda_{\max }$ can be used at all levels. In the present implementation, it is assumed that if $\lambda_{\max } / \lambda_{\min }>1.5$ then there is reasonable variation in the panel lengths. When splitting a cell $C$, into four daughter cells, the $\lambda_{\max }$ of cell $C$ is set as the $\lambda_{\max }$ of the panels in the children. When splitting the newly created daughter cells, if the cell size $h$ is such that $h<2 \beta \lambda_{\max }$, then $\lambda_{\max }$ is recomputed for the cell. After re-computation of $\lambda_{\max }$, if $h>2 \beta \lambda_{\max }$ then the cell can be split. If not the split cell will be smaller than the required length and is not split. The factor 2 arises because the split cell's length will be $h / 2$. Using such a scheme, the need to compute the maximum panel length at each level is eliminated and is computed only when necessary.

\section{Results and discussion.}

5.1. Influence of cell size on accuracy. Consider a panel placed at the edge of a cell as shown in figure 5.1. The cell $C$ contains a single panel at one corner such that the mid-chord of the panel is just inside it. The cells, $L 1, L 2$ and $L 3$ are well separated from $C$. The multipole expansion due to $C$ is evaluated on these cells. The multipole expansion of $C$ is also transferred to these cells as a local expansion and this local expansion is evaluated inside these cells. The contours of the relative error in the velocity due to the panel in these cells is plotted for the presently developed method, Anderson's scheme [1] and the scheme of Ramachandran et al. [11. As would be expected, it is seen that the point $z_{m}$ as shown in the figure has the maximum error. The cells $X 1$ and $X 2$ are cells which are in the $W_{b}$ list of $C$. Hence, $C$ is in the $X_{b}$ list of $X 1$ and $X 2$. The $\Delta_{b}$ interactions on these two are evaluated from cell $C$. In this case the maximum error occurs at the point shown as $z_{x}$ in figure 5.1. Again this is to be expected because this point is closest to the circle centered at $C$ and containing the panel completely. 


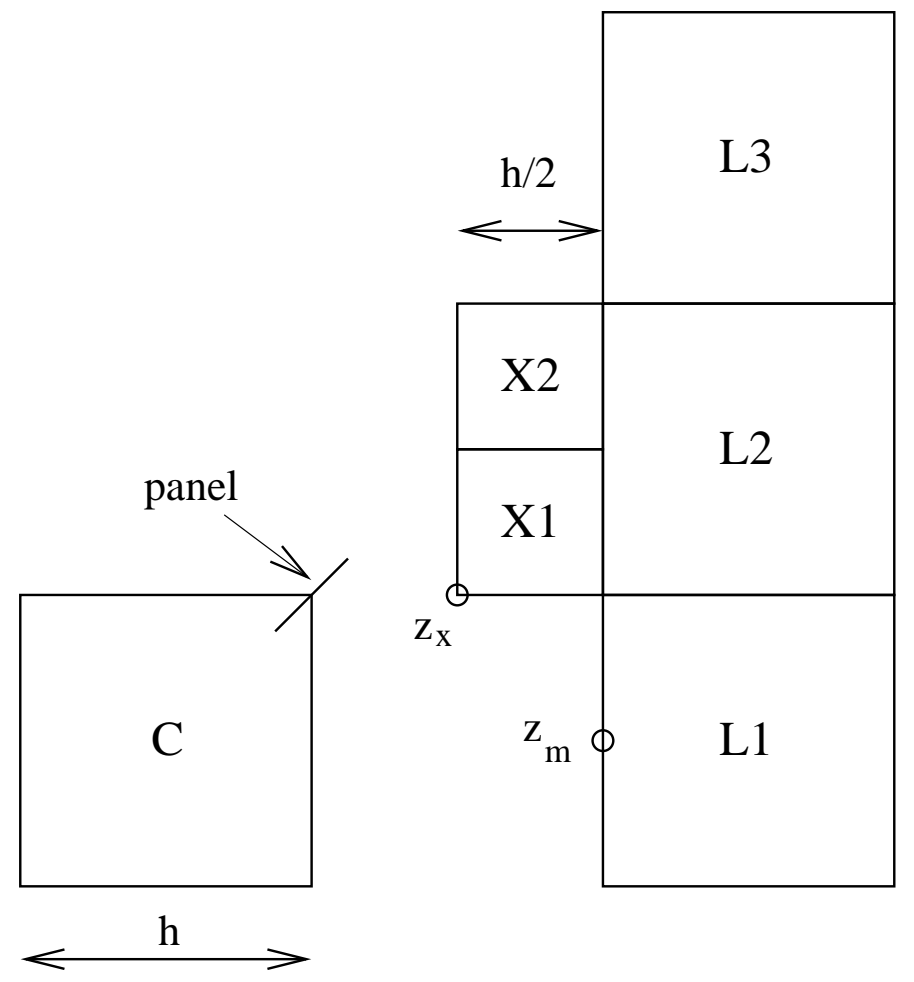

FIG. 5.1. Illustration of a panel in a cell and the location of maximum error. The cell $C$ contains a panel at the corner. The cells $X 1$ and $X 2$ are cells where the $\Delta_{b}$ evaluation is performed, $L 1, L 2, L 3$ are cells where local expansions and multipole expansions due to $C$ are computed.

The relative error, $E_{r e l}$, at a point is defined as,

$$
E_{\text {rel }}=\left|\frac{v_{\text {exact }}-v_{\text {computed }}}{v_{\text {exact }}}\right|
$$

Note that $v_{\text {exact }}$ is never zero at the points being considered. For a panel of fixed chord length, $\lambda$, different cells, $C$, with length given as $h=\beta \lambda, \beta>1$ are considered. For each of these cases the maximum relative error inside the cells $X 1$ and $L 1$, which are at the points $z_{x}$ and $z_{m}$ respectively, are computed. The number of terms, $p$, necessary to obtain an accuracy of around $10^{-6}$ are considered for the computation. At $z_{m}$ both the multipole expansion due to $C$ and the local expansion due to $L 1$ are computed. At $z_{x}$ the $\Delta_{b}$ interaction due to $C$ is computed. Figure 5.2 plots these errors as $\beta$ is varied. At the chosen scale, the local expansion error curve is indistinguishable from the multipole expansion error curve. This occurs because the order of the local expansion error is independent of $\beta$ as seen in equation ( 2.25$)$. Therefore, the local expansion is as inaccurate as the multipole expansion that was used to compute its coefficients. Figure 5.3 plots the error when Anderson's scheme is used. Figure 5.4 plots the error for the linear panel technique of Ramachandran et al.[11]. In this case a linear (not cubic) panel is used in order to make a fair comparison. This is because the method was developed for linear panels. Here also the local expansion error curve is indistinguishable from the multipole expansion error curve. As is expected, good accuracy is obtained only when $\beta>5$ for all the methods. 


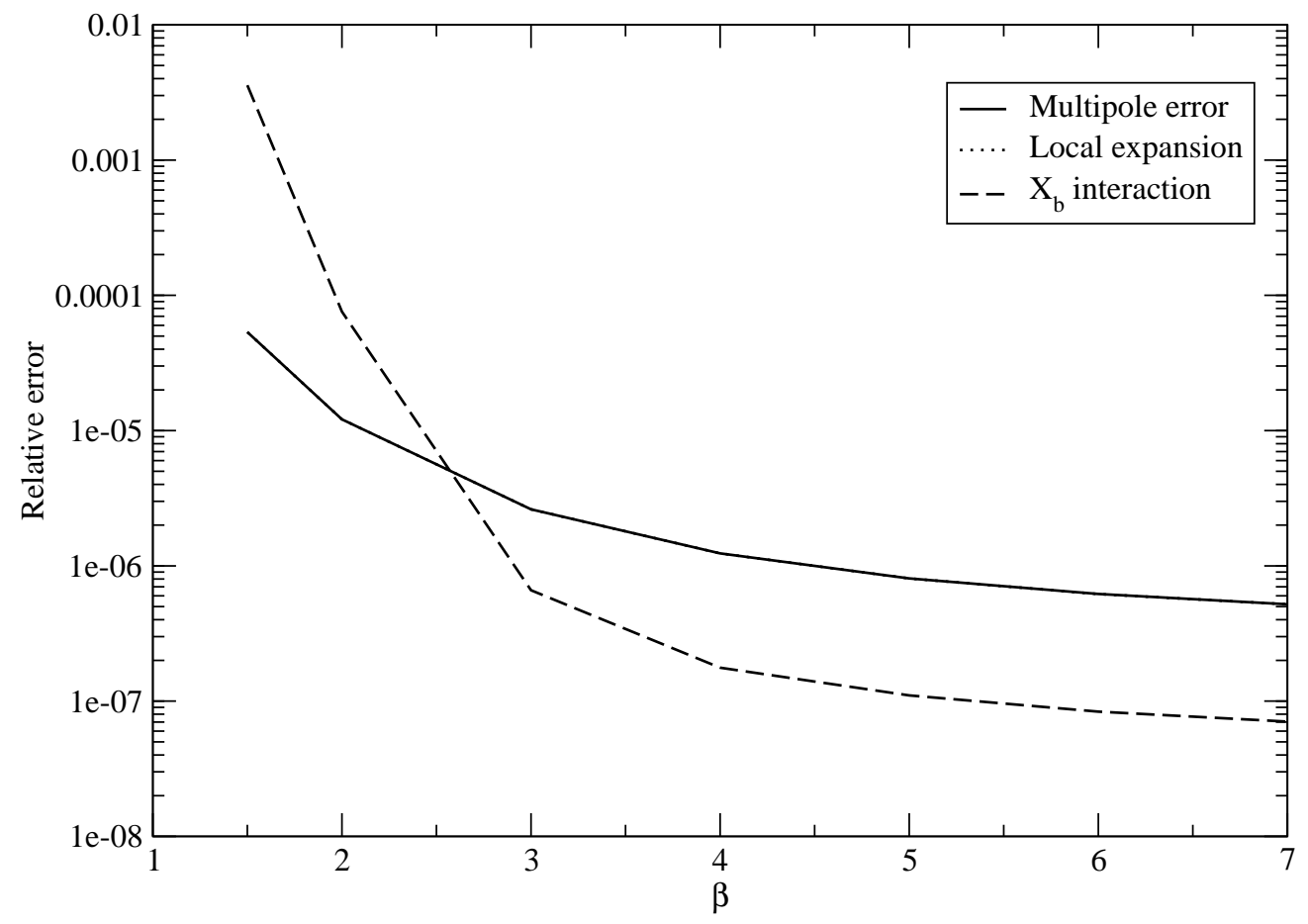

FIG. 5.2. Maximum relative error due to the multipole expansion, local expansion and $X_{b}$ interaction versus change in $\beta$ for the presently developed method. Note that the curves for the multipole expansion and the local expansion coincide.

This illustrates the importance of limiting cell size based on panel length.

5.2. Comparison of accuracy. In order to demonstrate the accuracy of the present method, the flow past a circular cylinder is used for comparison. This problem is chosen because it has an exact solution, the geometry is simple and has bounded values of vorticity. The flow past a circular cylinder of radius 1 unit with 400 cubic panels is considered. A circular ring of 10000 particles around the cylinder is considered at various distances from the surface of the cylinder. The boundary value problem is solved by using an LU decomposition. Once the singularity distribution on the panels is known, the velocity due to the panels is computed using the direct method (for cubic panels), the hybrid cubic/flat fast multipole panel approach developed in [11, the presently developed scheme and Anderson's 1] scheme. The relative error at each evaluation point is computed using equation (5.1). The maximum of these is plotted as the ring radius is varied in figure 5.5. It is to be noted that when the exact velocity is very small, the point is not considered for the error computation. For the scale used in the graph, the results for the direct method, the presently developed algorithm and Anderson's scheme all coincide. This indicates that the presently developed technique produces very accurate results.

5.3. Comparison of computational efficiency. A few general observations are made first. If the causes and effects in the domain decomposition are well separated 


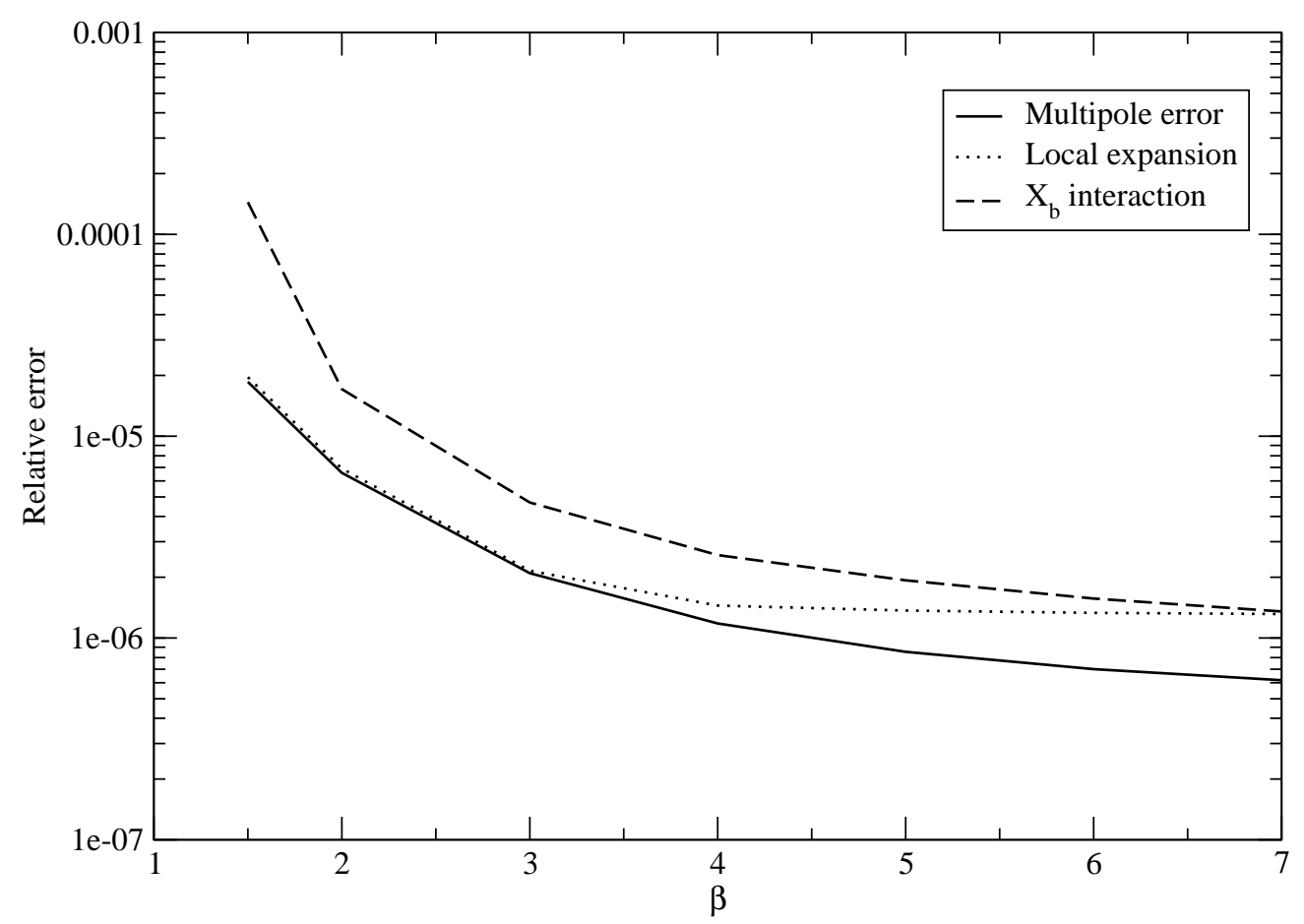

FIG. 5.3. Maximum relative error due to the multipole expansion, local expansion and $X_{b}$ interaction versus change in $\beta$ for Anderson's technique.

then the interactions between these causes and effects can be evaluated without direct computations. It is easy to see that this is the most efficient case. For example, consider the flow past a circular cylinder. Let the velocity or potential due to this cylinder be computed at a cluster of particles. If the cluster is at least two diameters away from the center of the cylinder, then there are no direct computations to perform between these particles and the panels that represent the cylinder. Clearly such a computation would be highly efficient. On the other hand if the cluster is such that there are a large number of particles that are distributed along the surface of the cylinder, then there are a large number of direct computations to be performed. Given that the size of a cell is restricted by the length of the panels (section [5.1), it is easy to imagine situations where a small number of panels are used along with a large number of particles distributed near them. In such a case, due to the size of the cell there will be a very large number of effect particles per cell. This will make the multipole computations inefficient. In order to overcome this one should reduce the size of the panels such that the ratio of the number of effects divided by the number of causes in a cell are kept as small as possible. So, on the one hand the number of panels must increase and on the other hand the main reason why higher order panels are chosen is to use a small number of panels. By using a small number of panels the matrix used to solve for the singularity distribution is small and hence easier and faster to solve. Given such a conflicting requirement it is still possible to use a smaller matrix 


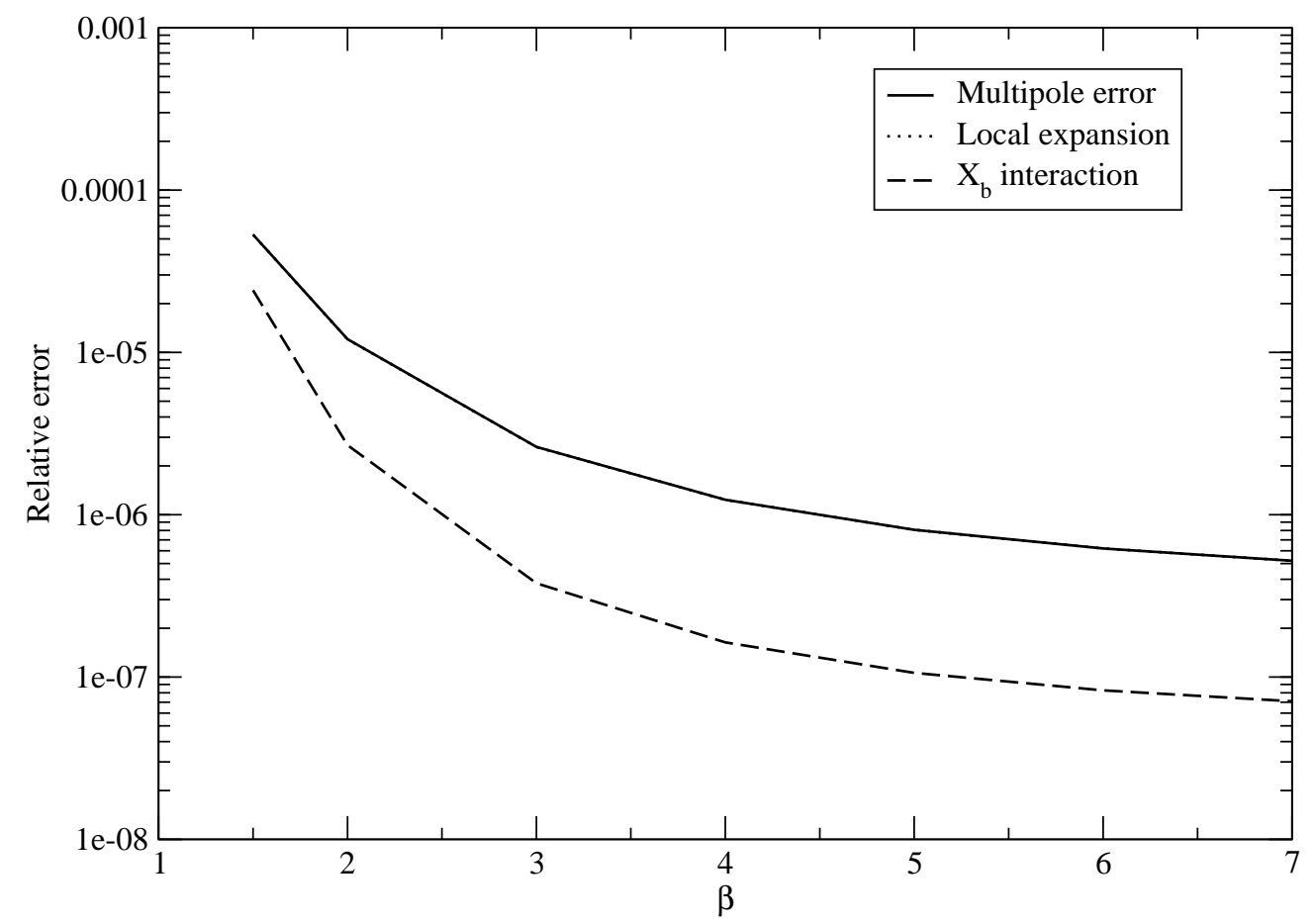

FIG. 5.4. Maximum relative error due to the multipole expansion, local expansion and $X_{b}$ interaction versus change in $\beta$ for the linear fast panel technique of Ramachandran et al. [11]. A linear panel is used for this computation. Note that the curves for the multipole expansion and the local expansion coincide.

by using two different representations for the body. One representation with larger panels could be used to solve for the singularity strengths and another representation with smaller panels, having interpolated strengths obtained from the larger panels, used for the FMM. This way it should be possible solve the boundary value problem for the panel singularities efficiently and also perform the FMM efficiently.

In order to demonstrate the efficiency of the FMM for higher order panels, the flow past a circular cylinder of radius 1 and centered at the origin is considered. The cylinder is discretized into 400 cubic panels. A uniform grid of particles in the square region $z_{\min }=-2.0-2.0 i, z_{\max }=2.0+2.0 i$ is considered. The number of particles used for the grid is varied. The time taken by the direct method, the hybrid flat/cubic FMM of Ramachandran et al. 11, the presently developed method and Anderson's algorithm are plotted in figure 5.6. The maximum number of cause and effects are chosen as 13 and 13 respectively for Anderson's scheme and 7 and 7 for all the others. $\beta$ is chosen as 7.0. As can be seen, all the fast multipole methods are significantly (factor of 50) faster than the direct method. It is also seen that the present scheme is slightly faster Anderson's scheme.

The computations have demonstrated that the developed method efficiently computes the velocity due to the panels on the passive particles. The boundary value problem for the panel method is solved using an LU decomposition. As explained in 


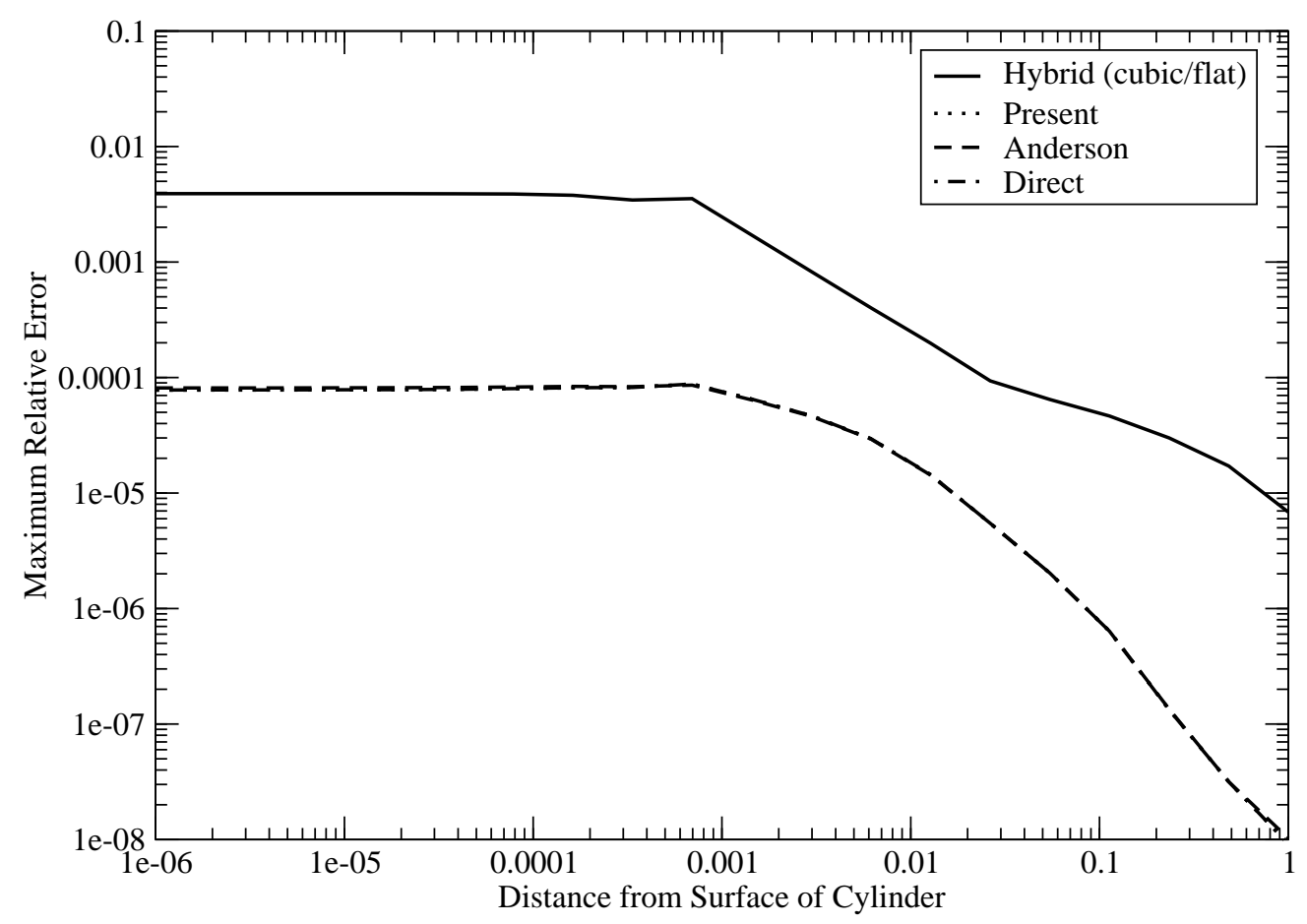

FIG. 5.5. Plot of the maximum relative error due to various methods versus distance from the surface of the cylinder. 400 panels are used for the cylinder. The curves for the presently developed method and Anderson's scheme coincide with the curve for the direct computation.

the introduction, the boundary value problem can be solved by an iterative method like the GCRA used by Rokhlin [12. While it is not explicitly demonstrated here, it is easy to see that the iterative method can be efficiently applied here by using the present method to evaluate the velocity of the panels.

It is to be noted that the computations for the above results are not entirely efficient because the number of panels and the grid size is fixed whereas the number of particles is increasing. This results in a very large number of effects per cell. In spite of this, the fast multipole schemes are much faster than the direct method.

An estimate for the number of effects per cell can be obtained in the following manner. Given the radius of the circle $r, \beta$, and the number of panels, $N_{\text {panel }}$, the length of the smallest cell is clearly limited by $h=2 \pi r \beta / N_{\text {panel }}$. If a uniform grid of $N_{p}$ particles enclosed in an area $A$ is used, then the estimated number of particles in the smallest cell, $n_{e}$, is

$$
n_{e}=\frac{N_{p}}{A} h^{2}=\frac{4 N_{p} \pi^{2} r^{2} \beta^{2}}{A N_{\text {panel }}^{2}}
$$

In order to demonstrate how significantly the number of effects per cell affects the computational time, the time taken for the presently developed algorithm for a fixed number of particles is computed as $n_{e}$ is changed. For a given $N_{p}, \beta$ and a given body, 


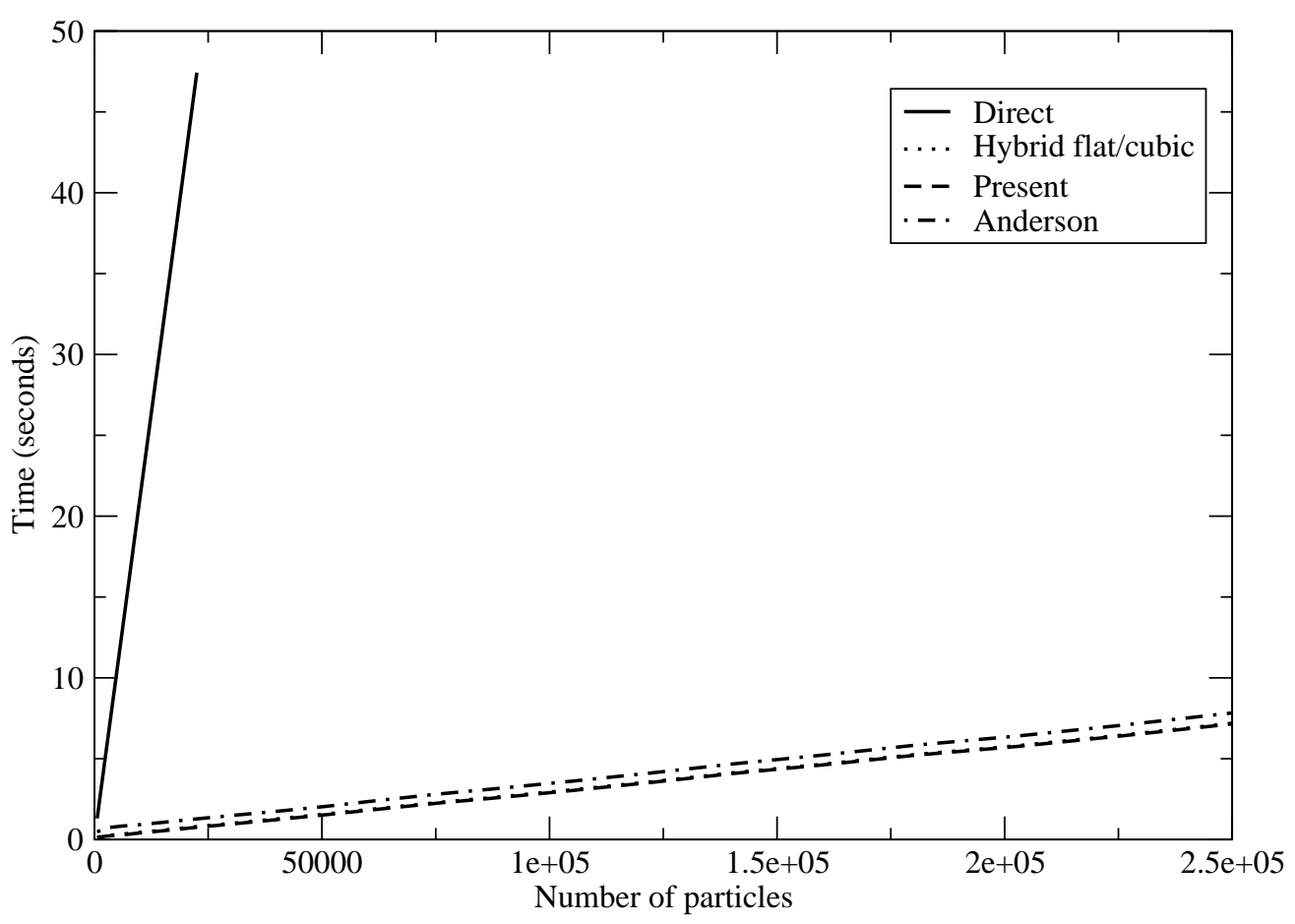

FIG. 5.6. Time taken versus number of particles on a square region. 400 panels are used for the cylinder. $\beta=7.0$. Note that the curves for the present method and the hybrid method of Ramachandran et al. [11] almost coincide.

$n_{e}$ can be varied by changing either $A$ (the area of the uniform grid of particles) or by changing $N_{\text {panel }}$.

Figure 5.7 plots the variation of the time taken as the area of the uniform grid is gradually increased. The number of tracer particles in the grid is 40000 . The stair stepping occurs in the plot because the cell size is limited by $\beta \lambda$ and the way in which the cells are split. If the total length of the side of a cell at level 0 is $L$, then the length of the side of the cell at level $l$ is, $L / 2^{l}$. In the range $9<n_{e}<11$, the length $L$ of the level 0 cell and the panel length are such that it is possible to refine the grid to one more level. This is the reason for the stair stepping in the plot. However, the point being made here is that as the number of effects per smallest cell reduces the time taken reduces significantly anywhere between a factor of 4 to 8 . It is also seen that for most part, the present scheme is about 1.5 to 2 times faster than Anderson's scheme.

Figure 5.8 plots the variation of the time taken as the number of panels is increased. 40000 tracer particles are considered inside the square region $\left(z_{\min }=\right.$ $\left.-2.0-2.0 i, z_{\max }=2.0+2.0 i\right)$ for all the computations. As can be seen from equation (5.2),$n_{e}$ drops as the number of panels increases. The time taken drops first and then increases as the number of panels is increased because initially the ratio of the length of the cell and the panel size is such that the first increase in the number 




FIG. 5.7. Time taken as $n_{e}$ is varied. $n_{e}$ is reduced by increasing A. 400 panels are used for the cylinder. $\beta=7.0$.

of panels triggers a split in the cell. Subsequently, reducing the panel length does not trigger a split until a threshold is crossed. During this time the computational time increases. As can be seen in the figure, even though the number of panels has increased by a factor of 16 the time taken to perform the computation has reduced by a factor of 4 . This illustrates the importance of choosing the right number of panels.

The time taken to re-compute the multipole coefficients (equation (2.8)) for each panel is small compared to the other computations. In the figure 5.8 the fraction of time taken to recompute the multipole is around $8 \%$ of the total time when the number of panels were 1600 . With 400 panels the time taken to re-compute the multipoles is around $1.5 \%$ of the total time. This indicates that the present scheme is efficient even if the panel geometry changes significantly in time.

It is to be noted that Anderson's scheme has been implemented with some care. The only difference between the newly developed algorithm and the implementation of Anderson's method is in the functions governing the computation of the multipoles at the finest level, transfer of multipole expansion and evaluation and transfer of the local expansion. The expressions given in Anderson's work are used in these functions. The velocity field is computed by analytically differentiating these expressions. All quantities that are constant (like $\log (a)$, where $a$ is the radius of the ring and the various sines and cosines) are pre-computed and stored in order to avoid unnecessary re-computation. It is also to be noted that the evaluation of the stream function 


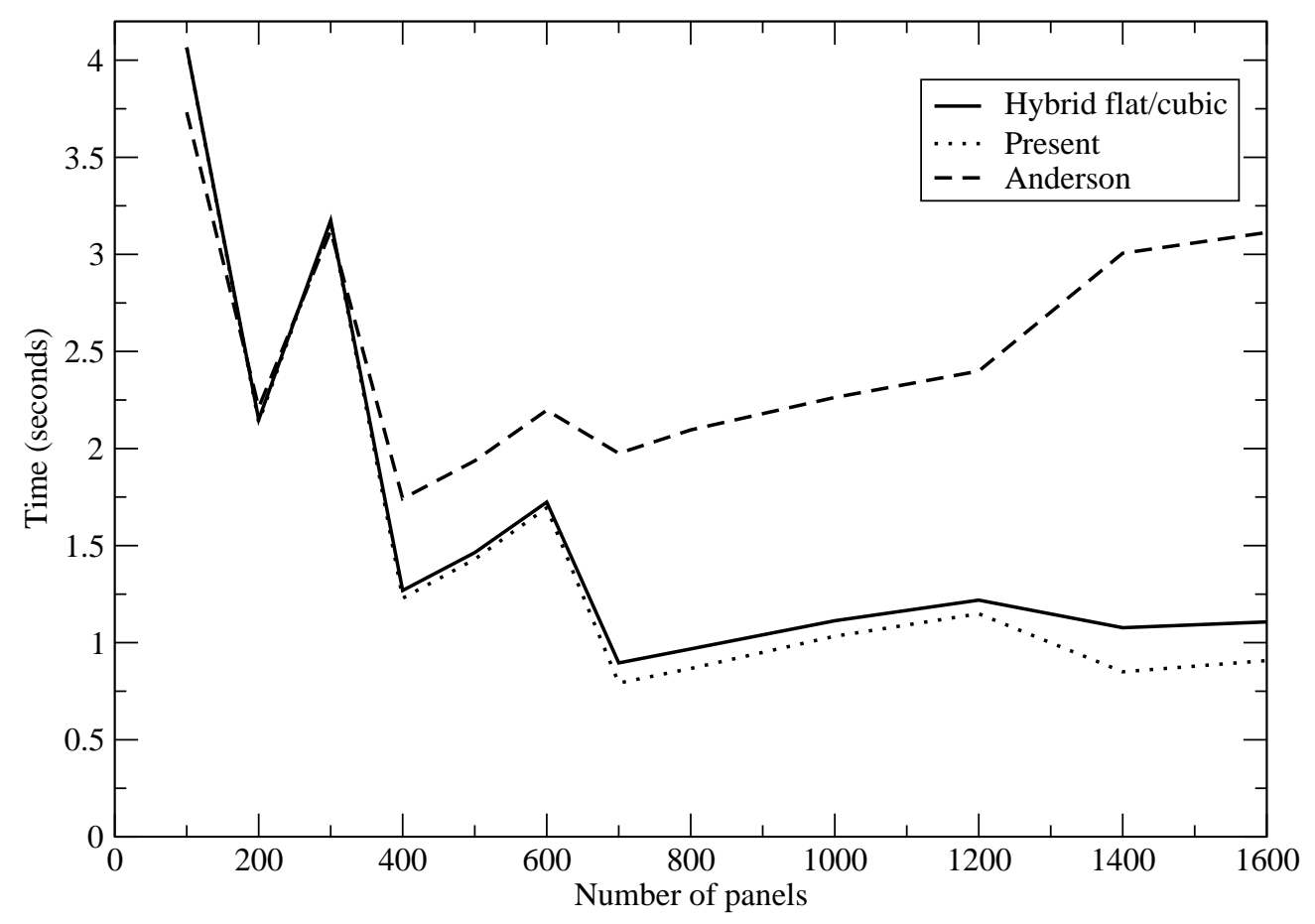

FIG. 5.8. Time taken as the number of panels is increased. 40000 points in a uniform grid are considered inside a square region. $\beta=7.0$.

did not amount to more than $5 \%$ of the total computational time taken to evaluate the velocity on a 101x101 grid of points. It is possible that our implementation of Anderson's method can be further optimized. However, it must be noted that the expressions in equations (3.1) and (3.2) require the evaluation of the arctangent (to compute the angle $\theta$ ) and the various cosines. By precomputing $e^{i M s_{i}}$ and $e^{i s_{i}}$, it is possible to obtain the other cosine terms using arithmetic operations and the value of $e^{i \theta}$ and $e^{i M \theta}$. However, the multipole expansions derived in the present work require purely arithmetic operations. Therefore, it appears that there is a small price to pay for the generality that Anderson's scheme provides and the ease of its deployment and use in different situations. The gain in using the present algorithm over Anderson's scheme depends on the choice of parameters and the problem chosen. Our implementation suggests that a factor of two improvement is possible.

6. Conclusions. The adaptive fast multipole method [2] has been successfully applied to higher order vortex panels. This has been demonstrated for panels with cubic geometry and linear vorticity distribution. Comparisons of accuracy and speed with the technique developed by Ramachandran et al. [11] and with Anderson's fast multipole method without multipoles [1] are very favorable. It is possible to extend an existing fast multipole code to use the new method. The paper also implements Anderson's technique in the framework of an adaptive fast multipole method. It is seen that although Anderson's method is much easier to implement it is slightly 
slower than the presently developed technique. The paper also shows that if accuracy is desired, the length of the smallest cell is to be restricted based on the size of the panels.

\section{REFERENCES}

[1] C. R. Anderson, An implementation of the fast multipole method without multipoles, SIAM J. Sci. Stat. Comput., 13 (1992), pp. 923-947.

[2] J. Carrier, L. Greengard, and V. Rokhlin, A fast adaptive multipole algorithm for particle simulations, SIAM J. Sci. Stat. Comput., 9 (1988), pp. 669-686.

[3] L. Greengard and V. Rokhlin, A fast algorithm for particle simulations, Journal of Computational Physics, 73 (1987), pp. 325-348.

[4] J. L. Hess And A. M. O. Smith, Calculation of potential flow about arbitrary bodies, Progress in Aeronautical Science, 8 (1966), pp. 1-138.

[5] J. Katz and A. Plotkin, Low-Speed Aerodynamics: From Wing Theory to Panel Methods, McGraw-Hill Education, New York, 1991.

[6] R. I. Lewis, Vortex Element Methods for Fluid Dynamical Analysis of Engineering Systems, Cambridge University Press, Cambridge, UK, 1991.

[7] J. Makino, Yet another fast multipole method without multipoles — pseudo-particle multipole method, Journal of Computational Physics, 151 (1999), pp. 910-920.

[8] E. MARTENSEn, The calculation of the pressure distribution on a cascade of thick airfoils by means of Fredholm integral equation of the second kind, NASA TTF, 702 (1971).

[9] W. H. Press, S. A. Teukolsky, W. T. Vetterling, and B. P. Flannery, Numerical Recipes in ' $C$ ': The art of scientific computing, Cambridge University Press, Cambridge, UK, 1992.

[10] P. Ramachandran, S. C. Rajan, and M. Ramakrishna, An accurate two-dimensional panel method, in Seminar on advances in aerospace technologies, SAAT-2000, Aeronautical Society of India, January 2000.

[11] - A fast, two-dimensional panel method, SIAM Journal on Scientific Computing, 24 (2003), pp. 1864-1878.

[12] V. Rokhlin, Rapid solution of integral equations of classical potential theory, Journal of Computational Physics, 60 (1985), pp. 187-207.

[13] P. W. C. Vosbeek, H. J. H. Clercx, And R. M. M. MattheiJ, Acceleration of contour dynamics simulations with a hierarchical-element method, Journal of Computational Physics, 161 (2000), pp. 287-311. 